\title{
The Effect of Nutritive and Non-Nutritive Sweeteners on the Growth, Adhesion, and Biofilm Formation of Candida albicans and Candida tropicalis
}

\author{
Manjula M. Weerasekera ${ }^{a}$ Thilini A. Jayarathna ${ }^{a}$ Gayan K. Wijesinghe \\ Chinthika P. Gunasekara ${ }^{a}$ Neluka Fernando ${ }^{a}$ Nilwala Kottegoda ${ }^{b-d}$ \\ Lakshman P. Samaranayake ${ }^{\mathrm{e}}$
}

${ }^{a}$ Department of Microbiology, Faculty of Medical Sciences, ${ }^{b}$ Center for Advanced Material Research, and ${ }^{\mathrm{C}}$ Department of Chemistry, Faculty of Applied Sciences, University of Sri Jayewardenepura, Nugegoda, and d Sri Lanka Institute of

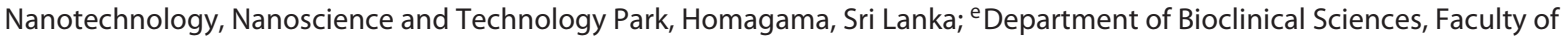
Dentistry, Kuwait University, Kuwait City, Kuwait

\section{Significance of the Study}

- In this study, the non-nutritive sweetener saccharin dampened the in vitro biofilm growth of both Candida albicans and Candida tropicalis. The finding revealed that saccharin could suppress yeast growth and biofilm formation within the oral milieu and hence indirectly contribute to oral health.

\section{Keywords}

Candida species · Biofilms · Adhesion · Growth sucrose · Glucose $\cdot$ Saccharin

\begin{abstract}
Objective: To determine the effect of glucose, sucrose, and saccharin on growth, adhesion, and biofilm formation of Candida albicans and Candida tropicalis. Materials and Methods: The growth rates of mono-cultures of planktonic C. albicans and C. tropicalis and 1:1 mixed co-cultures were determined in yeast nitrogen broth supplemented with $5 \%$ (30 $\mathrm{mm}$ ) and 10\% (60 mm) glucose, sucrose, and saccharin, using optical density measurements at 2-h intervals over a 14-h period. Adhesion and biofilm growth were performed and the growth quantified using a standard 3-(4,5-dimethylthiazol-2-yl)-2,5-diphenyltetrazolium bromide (MTT) assay. The biofilm architecture was visualized using scanning electron microscopy. One- and two-way analysis of variance
\end{abstract}

\begin{tabular}{ll}
\hline KARGER & $\begin{array}{l}\text { ( } 2017 \text { The Author(s) } \\
\text { Published by S. Karger AG, Basel }\end{array}$ \\
$\begin{array}{l}\text { E-Mail karger@karger.com } \\
\text { www.karger.com/mpp }\end{array}$ & $\begin{array}{l}\text { This is an Open Access article licensed under the Creative Commons } \\
\text { Attribution-NonCommercial-4.0 International License (CC BY-NC) } \\
\text { (http://www.karger.com/Services/OpenAccessLicense), applicable to } \\
\text { the online version of the article only. Usage and distribution for com- } \\
\text { mercial purposes requires written permission. }\end{array}$
\end{tabular}

(ANOVA) was performed to analyse the differences among multiple means. Results: The highest planktonic growth was noted in $5 \%$ glucose after $14 \mathrm{~h}(p<0.05)$. No significant planktonic growth was observed in either concentration of saccharin. Both the concentrations of glucose and sucrose elicited significantly increased adhesion from MTT activity of 0.017 to $>0.019$ in mono- as well as co-cultures $(p<0.05)$, whilst the lower concentration of saccharin significantly dampened the adhesion. Maximal biofilm growth was observed in both species with the lower concentration of sucrose $(5 \%)$, although a similar concentration of saccharin abrogated biofilm development: the highest MTT value $(>0.35)$ was obtained for glucose and the lowest ( $>0.15)$ for saccharin. Conclusion: In this study, glucose and sucrose accelerated the growth, adhesion, and biofilm formation of Candida species. However, the non-nutritive sweetener saccharin appeared to dampen, and in some instances suppress, these virulent attributes of Candida.

(c) 2017 The Author(s)

Published by S. Karger AG, Basel
Manjula M. Weerasekera

Department of Microbiology, Faculty of Medical Sciences

University of Sri Jayewardenepura, Gangodawila

Nugegoda 10250 (Sri Lanka)

E-Mailmmweera@sjp.ac.lk, or mmweera@yahoo.com 


\section{Introduction}

Candida species are opportunistic fungal pathogens that inhabit $50-60 \%$ of the oral cavities of humans [1]. Although Candida albicans is the predominant human oral commensal, other species such as Candida tropicalis are also commonly isolated from the oral niche $[2,3]$. Indeed, it had been reported that $10-15 \%$ of oral candidal infections are due to mixed Candida species, in particular C. albicans and C. tropicalis [4]. These fungi cause frequent recalcitrant oral and systemic infections particularly in compromised patient populations such as those with HIV infection and patients on immunosuppressive therapy [5].

Although a yeast of low virulence, Candida species possess a range of pathogenic mechanisms, including the ability to form complex biofilms either with other communal bacteria or yeasts on mucosal and tooth surfaces or on acrylic denture surfaces [6, 7]. Both C. albicans and C. tropicalis are known to produce extensive oral biofilms both in vitro and in vivo, and some are macroscopically visible as white, pseudo-membranes in disease such as oral thrush [8-10]. Furthermore, it is known that fermentable dietary sugars including sucrose and glucose promote Candida growth, adhesion, and biofilm development [11] and could aggravate oral diseases, in particular Candida-associated denture stomatitis and dental caries [12].

Sucrose is a naturally occurring, universally available, and widely consumed, yet highly cariogenic, dietary sugar [13]. It serves as a substrate for the synthesis of extracellular and intracellular polysaccharides of plaque biofilms [13]. The monosaccharide glucose, a by-product of sucrose fermentation, is also known to contribute equally to the development of oral biofilms [13]. Due to the diverse health concerns associated with rampant and worldwide sucrose consumption, an array of non-nutritive sweeteners such as saccharin, sucralose, aspartame, acesulfame-K, and neotame $[14,15]$ have been introduced in the food industry and are widely available. Non-nutritive sweeteners are known as chemosensory signalling compounds that influence digestive processes and behaviour [16]. Saccharin is by far the most widely consumed nonnutritive sweetener and is commonly recommended for patients with diabetes as an alternative to the fermentable sugar sucrose [16]. Most of the commercial toothpastes and mouth washes also contain a percentage of non-nutritive sweeteners, especially saccharine $[17,18]$.

Both nutritive and non-nutritive sweeteners are extensively consumed worldwide $[15,16]$. Different environ- mental conditions such as nutritional sources can influence the activities of micro-organisms. The type of sugar and the concentration are two of the major determinants of biofilm activity of Candida species in the oral cavity [11]. The effect of both fermentable natural and non-fermentable synthetic sugars on oral biofilms should be investigated because of the increased consumption of these sugars and artificial sweeteners. Their relative impact on the growth, adhesion, and biofilm development of the opportunistic oral fungal pathogen Candida has not been studied. Hence, the objective of this study was to investigate in vitro the effect of 5 and 10\% sucrose, glucose, and saccharin on biofilm formation of C. albicans and C. tropicalis.

\section{Materials and Methods}

\section{Candida Strains and Planktonic Growth}

C. albicans and C. tropicalis (ATCC ${ }^{\circledR} 10231$ and 13803, respectively) derived from the American Type Culture Collection [19] were used in this study. After confirmation of their identity by using a germ tube test (culture on CHROM agar and cornmeal Tween 80 agar), the yeasts were subcultured on Sabouraud dextrose agar (Sigma-Aldrich, USA) and incubated at $35^{\circ} \mathrm{C}$ for $48 \mathrm{~h}$. The resultant growth was harvested and subcultured in yeast nitrogen broth (YNB; Sigma-Aldrich) supplemented with either 30 $\mathrm{mm}(5 \%)$ or $60 \mathrm{~mm}(10 \%)$ glucose, $15 \mathrm{~mm}(5 \%)$ or $30 \mathrm{~mm}(10 \%)$ sucrose, or $25 \mathrm{~mm}(5 \%)$ or $50 \mathrm{mM}(10 \%)$ sodium saccharin (all Sigma Aldrich). Controls were prepared in YNB medium without adding sugars.

Planktonic growth rates of the yeasts were determined using the method initially described by Jin et al., $[4,11]$ with minor modifications. Briefly, suspensions of C. albicans and C. tropicalis $\left(10^{6}\right.$ cells/mL) mono-cultures, and a co-culture of the yeasts (1:1 suspension; $10^{6}$ cells $/ \mathrm{mL}$ ) were prepared in YNB and supplemented with glucose, sucrose, or saccharin. A 100- $\mu \mathrm{L}$ sample of prepared Candida cell suspensions was inoculated in triplicate into a 96well, flat-bottom, microtiter plate. The growth rates were then determined by optical density $\left(\mathrm{OD}_{492}\right)$ measurements in each well at $492 \mathrm{~nm}$ at 2-h intervals for $14 \mathrm{~h}$ using a microtiter plate reader (SpectraMax Plus 384; Molecular Devices, Inc., USA) and growth curves were prepared $[8,11]$.

\section{Candidal Adhesion Studies}

Standard cell suspensions $\left(10^{7}\right.$ cells $\left./ \mathrm{mL}\right)$ of C. albicans and C. tropicalis were prepared in sterile YNB laced with the different concentrations of glucose, sucrose, or saccharin. Both mono-species suspensions of $C$. albicans and C. tropicalis and a 1:1 mixture of C. albicans and C. tropicalis suspensions were prepared.

To examine the initial adhesion phase, $100 \mu \mathrm{L}$ of the prepared standard cell suspensions were inoculated in triplicate wells of a microtiter plate and incubated for $90 \mathrm{~min}$ at $37^{\circ} \mathrm{C}[6,8]$. After 90 min of incubation, the plates were carefully washed twice with 200 $\mu \mathrm{L}$ sterile PBS to remove non-adherent cells, and the adherent cells were quantified using a tetrazolium salt 3-(4,5-dimethylthiazol2-yl)-2,5-diphenyltetrazolium bromide (MTT) assay (Sigma-Aldrich) [20]. 


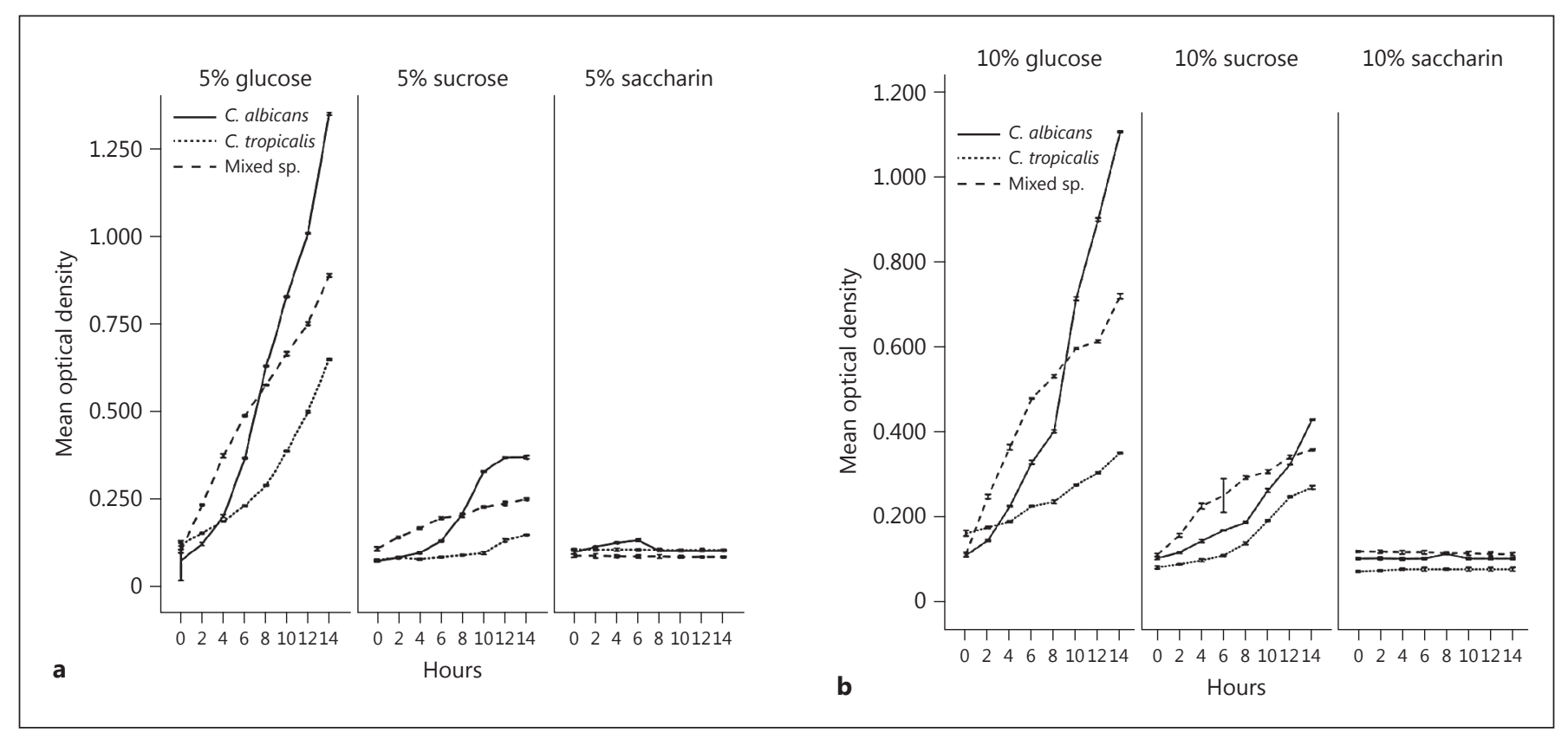

Fig. 1. a Growth curves of planktonic Candida albicans and Candida tropicalis and 1:1 mixed co-culture grown in glucose, sucrose, and saccharin 5\% (mean \pm 2 SD of 3 independent experiments performed in triplicate). b Growth curves of planktonic C. albicans and C. tropicalis and 1:1 mixed co-culture grown in glucose, sucrose, and saccharin $10 \%$ (mean \pm 2 SD of 3 independent experiments performed in triplicate).

For the MTT assay, $1 \mathrm{mg} / \mathrm{mL}$ aqueous solution of MTT was prepared. To quantify the adherent viable cell mass, $50 \mu \mathrm{L}$ of the latter solution was added to each well and incubated at $37^{\circ} \mathrm{C}$ for $4 \mathrm{~h}$. Afterwards, the MTT solution was carefully removed, $100 \mu \mathrm{L}$ of dimethyl sulfoxide (DMSO; Sigma-Aldrich) was added to solubilize the produced product, and absorbance was measured at wavelengths of 570 and $630 \mathrm{~nm}$.

\section{Candidal Biofilm Growth}

The growth rates of the biofilms were determined using the method described previously by Jin et al. $[4,11]$ with modifications. Standard cell suspensions $\left(10^{7}\right.$ cells $\left./ \mathrm{mL}\right)$ of $C$. albicans, $C$. tropicalis, and mixed species were prepared in sterile PBS, as described above, and $100 \mu \mathrm{L}$ was inoculated into wells of a microtiter plate in triplicate. The plates were incubated for $90 \mathrm{~min}$ at $37^{\circ} \mathrm{C}$ to initiate the adhesion phase. Then, the plates were washed twice with $200 \mu \mathrm{L}$ of sterile PBS and filled with $100 \mu \mathrm{L}$ of sterile YNB supplemented with the appropriate concentrations of the sweeteners. Subsequently, the plates were incubated at $37^{\circ} \mathrm{C}$ for $96 \mathrm{~h}$ and the culture medium was replenished daily. The biofilm biomass was quantified at 24, 48, 72, and $96 \mathrm{~h}$ using the MTT assay, and growth curves were constructed. In addition, $\mathrm{pH}$ values of the biofilms were measured before and after the addition of sugars.

\section{Scanning Electron Microscopy}

In order to examine the ultrastructural features, Candida biofilms were developed on 10-mm diameter glass coverslips using the identical procedure described above. The biofilms were fixed with $2.5 \%$ glutaraldehyde solution for $2 \mathrm{~h}$ and serially dehydrated with ethanol. After overnight drying in a desiccator, gold-coated biofilms were examined with scanning electron microscopy (SEM) (Hitachi SU 6600, Japan). The matured 1:1 mixed species (C. albicans and C. tropicalis) biofilm (72 h old) grown with the presence of $5 \%$ sucrose and C. albicans biofilm grown with the presence of $5 \%$ sucrose and $5 \%$ saccharin were visualized using SEM $[8,21$, 22]. These procedures were done in triplicate.

\section{Statistics}

One- and two-way analysis of variance (ANOVA) was performed to analyse the differences among multiple means using the Statistical Package for Social Sciences (SPSS) for windows, version 16.0 (SPSS Inc., Chicago, IL, USA) The $p$ value was used to determine the significant differences of adhesion among sugars. $p$ values $<0.05$ were considered statistically significant.

\section{Results}

\section{Candidal Growth}

The planktonic cultures of C. albicans and C. tropicalis exhibited copious growth in the two concentrations of both sugars (glucose and sucrose), although much more profuse growth was discernible in glucose. With 5\% glucose, $C$. albicans exhibited a maximum growth $(1.350$ $\left.\mathrm{OD}_{490}\right)$ after $14 \mathrm{~h}$ followed by $5 \%$ sucrose $\left(0.370 \mathrm{OD}_{490}\right)$ 


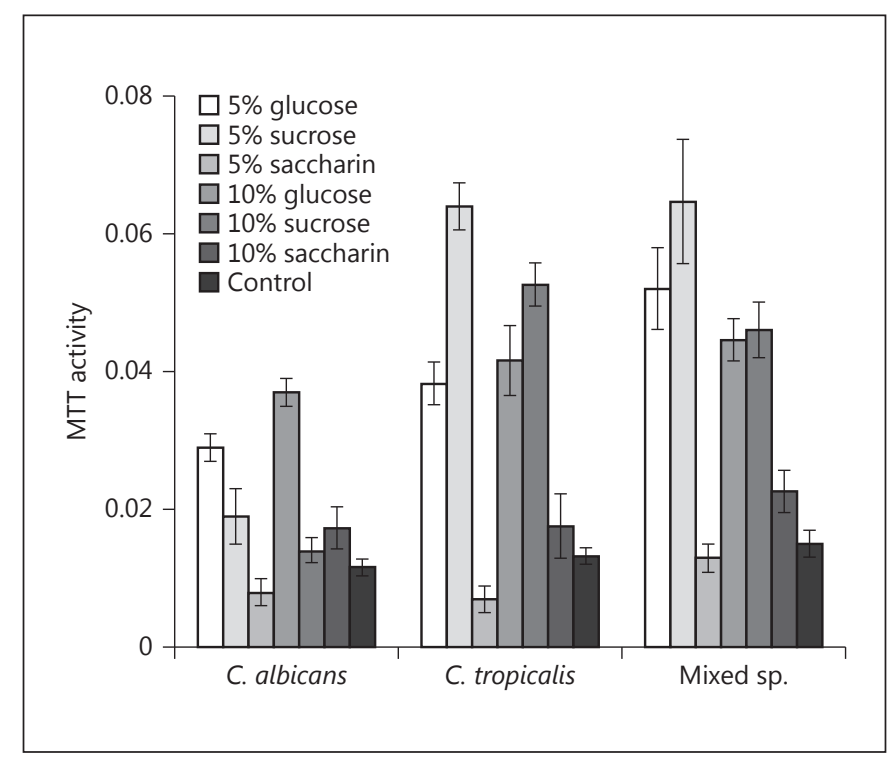

Fig. 2. Results of the MTT assay. Adhesion of C. albicans, C. tropicalis, and mixed species in yeast nitrogen broth (YNB) supplemented with 5 and $10 \%$ glucose, sucrose, and saccharin. In this experiment YNB without sugar was used as negative control. All error bars represent the mean $\pm 2 \mathrm{SD}$.

and $5 \%$ saccharine $\left(0.104 \mathrm{OD}_{490}\right)$. With $10 \%$ glucose, sucrose, and saccharin after $14 \mathrm{~h}$ the growth was recorded as $1.105,0.425$, and $0.100\left(\mathrm{OD}_{490}\right)$ respectively. C. tropicalis and mixes species also exhibited a similar growth pattern.

Saccharin did not support the planktonic growth of either the mono- or co-cultures of the two species. Both species showed continuous, uninterrupted growth and achieved a maximal cell density at $14 \mathrm{~h}$. In relative terms, the mono-culture growth of $C$. albicans was more intense than that of $C$. tropicalis, whilst the co-cultures demonstrated an intermediate growth profile (Fig. 1).

\section{Candidal Adhesion}

Compared to the sugar-free controls, $5 \%$ glucose and $5 \%$ sucrose (Fig. 2) exposure, either in mono- or co-culture, promoted the adhesion of both Candida species $(p<0.001)$. However, $5 \%$ saccharin exposure significantly reduced the adhesion of $C$. albicans and $C$. tropicalis compared to the saccharin-free control $(p<0.05)$, although no effect was noted in the co-cultures. An increased concentration of saccharin (10\%) promoted the adhesion in both the mono- and co-cultures of C. albicans $(p<0.005)$, but not in those of $C$. tropicalis $(p>$ $0.05)$.

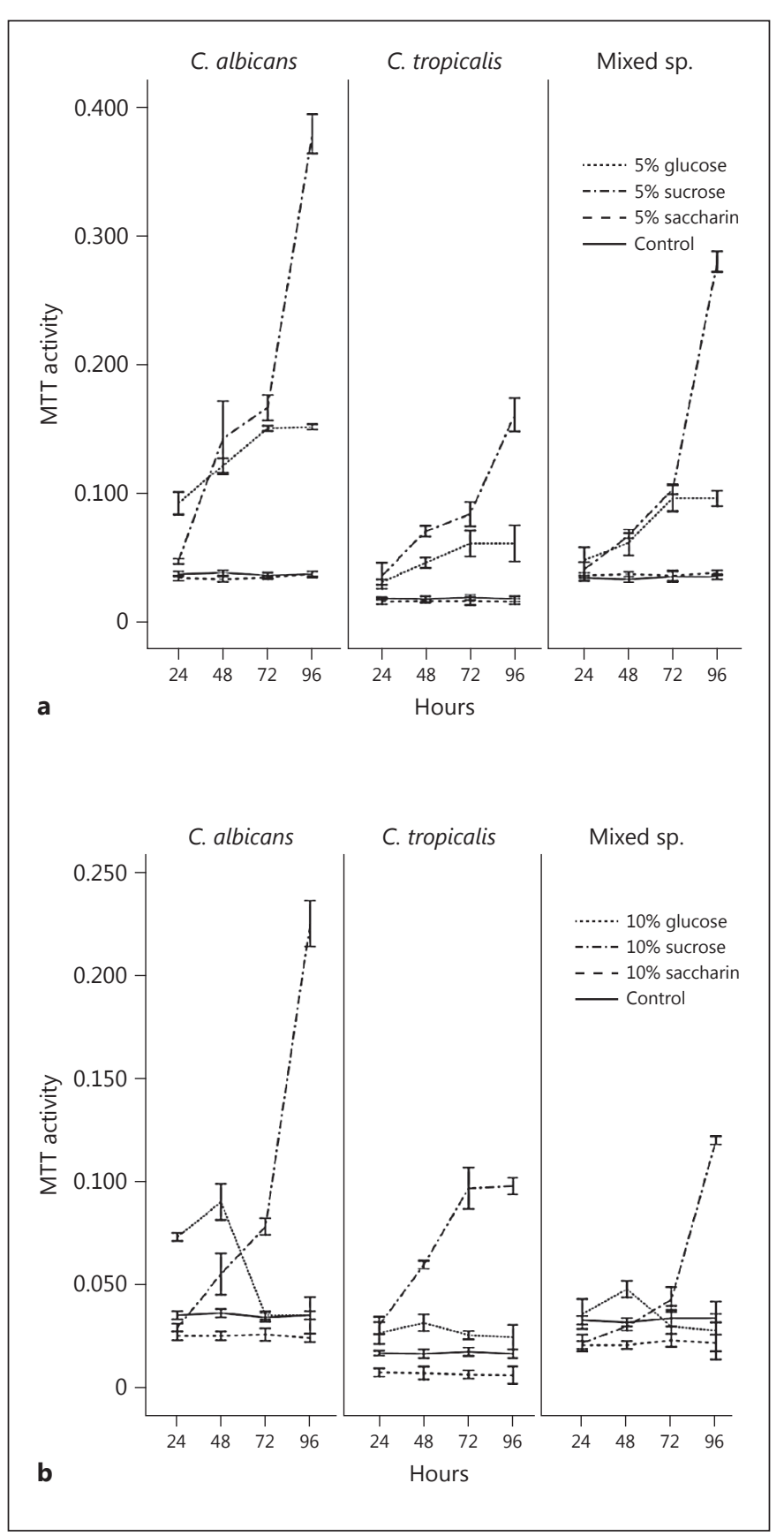

Fig. 3. a Biofilm development of C. albicans, C. tropicalis, and mixed species in yeast nitrogen broth (YNB) supplemented with $5 \%$ glucose, sucrose, and saccharin as determined by MTT assay (mean \pm 2 SD of 3 independent experiments performed in triplicate). b Biofilm development of C. albicans, C. tropicalis, and mixed species in YNB supplemented with $10 \%$ glucose, sucrose, and saccharin as determined by MTT assay (mean \pm 2 SD of 3 independent experiments performed in triplicate). 

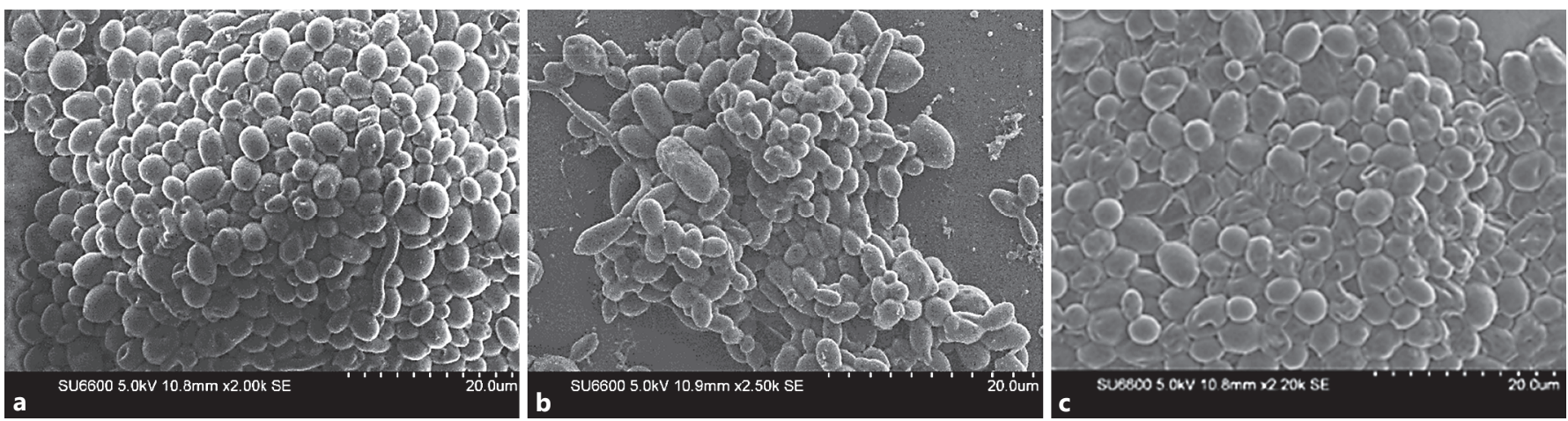

Fig. 4. a Electron microscopic appearance of 72 -h mature biofilms of C. albicans with $5 \%$ sucrose. Scale, $20 \mu \mathrm{m}$. b Electron microscopic appearance of 72-h mature biofilms of C. albicans and C. tropicalis mixed species coculture with $5 \%$ sucrose. Scale, $20 \mu \mathrm{m}$. c Electron microscopic appearance of 72-h mature biofilms of C. albicans with $5 \%$ saccharin. Scale, $20 \mu \mathrm{m}$.

\section{Candidal Biofilm Development}

In $5 \%$ sucrose suspensions, biofilm development in both the mono- and co-cultures of Candida was rapid with peak growth at $96 \mathrm{~h}$. The sweetener-free, control suspension was devoid of biofilm growth (Fig. 3a). A similar growth pattern was noted in $10 \%$ sucrose, except for $C$. tropicalis biofilm activity which appeared to plateau after 48 h (Fig. 3b). Saccharin did not support candidal biofilm development.

\section{Scanning Electron Microscopy}

The 72-h-old biofilms of $C$. albicans and the 1:1 mixed species biofilms of $C$. albicans and C. tropicalis grown with the presence of $5 \%$ sucrose were subjected to SEM to visualize the ultrastructure, as the highest growth was obtained with the respective concentration of sucrose. Further Candida biofilms grown for $72 \mathrm{~h}$ with the presence of $5 \%$ saccharin were also subjected to SEM as it showed a reduced growth. In a comparison of biofilm formation of C. albicans and C. tropicalis, C. albicans was the most prolific biofilm former based on the SEM observations.

The mature biofilm of $C$. albicans demonstrated a heavy aggregate of almost equal-sized yeast blastospores with very few hyphal elements (Fig. 4a). The co-culture mixed species biofilms had a similar structure, and the species were distinguishable by virtue of the marginally larger-sized C. tropicalis blastospores (Fig. 4b). In contrast, the biofilm of $C$. albicans in 5\% saccharin was less dense and devoid of either hyphal or budding blastospores. Extracellular polymeric material which is characteristic of biofilms was not visualized in any of the biofilms analysed (Fig. 4c).

\section{Discussion}

In this study, C. tropicalis adhesion was facilitated in both 5 and 10\% sucrose concentration, while C. albicans adhesion was promoted with both 5 and $10 \%$ glucose concentrations. Although these sugars facilitate the adhesion of the two species, the overall biofilm growth of $C$. albicans and C. tropicalis did not get promoted with $10 \%$ sucrose, glucose, or saccharine. In high concentrations of sugar, the water concentration is less, and this fact could act as a limiting factor for the biofilm growth of C. albicans and C. tropicalis. With the increase of sugar concentration in the medium, biofilm/micro-organism respiration and bio-synthesis rates will increase. However, in this situation water became a limiting factor due to the high sugar concentration in the surrounding media. These data are similar to previous findings of a number of researchers [23-25]. Both sugars are known to promote the production of a floccular, fibrillar layer, especially in C. albicans, which is thought to mediate adhesion and subsequent biofilm formation [26]. The adhesion of suspended, planktonic cells onto either a biotic surface such as the oral mucosa or an abiotic surface such as an acrylic denture is the initial step in biofilm formation.

Saccharin 5\% reduced adhesion of both C. albicans and C. tropicalis. However, $10 \%$ saccharin facilitated the adhesion of both Candida species. Saccharin had a paradoxical effect on yeast adhesions, as lower concentrations (5\%) of this non-nutritive sweetener reduced the adhesion of both yeast species whereas higher concentrations (10\%) facilitated the adhesion of both Candida species. The attachment of Candida species to the surface is medi- 
ated by electrostatic forces and certain other non-specific interactions. Further, the contribution of a high concentration of sodium in $10 \%$ saccharin could influence the electrostatic interactions between the cell surface and the polystyrene plate surface compared to other non-polar sugar molecules which promote candidal adhesion.

Fermentable sugars such as sucrose and glucose play a critical role in the initiation and development of major oral diseases such as caries and Candida-associated denture stomatitis. In an early study it was demonstrated that dietary carbohydrates, also termed nutritive sweeteners, including glucose and sucrose promoted adhesion and colonization of Candida species on both biotic and abiotic surfaces of the oral cavity [27]. Available data revealed that there is a strong relationship between quorum sensing, quorum sensing inhibition/quorum quenchers, and environmental and nutritional conditions including carbon source (type of sugar) [28]. Quorum sensing is a key contributory factor of microbial social behaviours and biofilm development. As regards planktonic growth of the yeasts, our data confirm the pervious findings that both glucose and sucrose promote yeast growth, and glucose is better at fostering such growth. Noteworthy, however, was the fact that saccharin did not support the planktonic growth of either the mono- or co-cultures of the two species, implying that the yeasts are incapable of metabolizing this substrate.

The finding that both glucose and sucrose facilitated candidal biofilm development confirmed the findings of a previous study [12]. Equally, the finding that C. albicans was a superior biofilm former to $C$. tropicalis corroborated previous findings [10] because Candida is a yeast which prefers to grow under low $\mathrm{pH}$ conditions, but $\mathrm{pH}$ 5 created by $10 \%$ sucrose did not foster its growth. Akin to planktonic cultures, saccharin did not support biofilm growth of either Candida species.

The co-culture studies of the two yeast species confirmed that they grew well together and had the ability to form multi-species biofilms. Clinical findings have also indicated that both C. albicans and C. tropicalis could cause poly-fungal infections in the oral cavity and elsewhere [29].

The ultrastructural findings of the biofilms confirmed the classic architecture of candidal biofilms. Extracellular polysaccharide material covering the biofilms (an integral biofilm component) was not visualized probably due to a processing artefact such as high vacuum and to the electron beam of SEM. Most importantly, the candidal cells were crenate, some demonstrating pockmarks with the presence of $5 \%$ saccharine, possibly indicating cell death due to loss of contents.

Clinically, these findings could imply that saccharin might also suppress yeast growth and biofilm formation within the oral milieu, and indirectly contribute to oral health.

\section{Conclusion}

Both glucose and sucrose accelerated the growth, adhesion, and biofilm formation of Candida species, either in mono- or co-cultures, to varying degrees. However, the widely used artificial, non-nutritive sweetener saccharin appeared to dampen, and in some instances suppress, these virulent attributes of Candida. Further studies are recommended to clarify the mechanism underlying this phenomenon, which has not been previously reported.

\section{References}

1 Samaranayake LP: Host factors and oral candidosis; in Samaranayake LP, MacFarlane TW (eds): Oral Candidosis. London, Butterworth, 1990, pp 66-103.

2 Sardi JC, Scorzoni L, Bernardi T, et al: Candida species: current epidemiology, pathogenicity, biofilm formation, natural antifungal products and new therapeutic options. J Med Microbiol 2013;62:10-24.

3 Weerasekera MM, Sissons CH, Wong L, et al: Use of denaturing gradient gel electrophoresis for the identification of mixed oral yeasts in human saliva. J Med Microbiol 2013;62: 319-330.
4 Jin Y, Yip HK, Samaranayake YH, et al: Biofilm-forming ability of Candida albicans is unlikely to contribute to high levels of oral yeast carriage in cases of human immunodeficiency virus infection. J Clin Microbiol 2003; 41:2961-2967.

5 Vazqhez JA: Optimal management of oropharyngeal and esophageal candidiasis in patients living with HIV infection. HIV AIDS (Aukl) 2010;2:89-101.

6 Thein ZM, Seneviratne CJ, Samaranayake $\mathrm{YH}$, et al: Community lifestyle of Candida in mixed biofilms: a mini review. Mycoses 2009; 52:467-475.
7 Weerasekera MM, Sissons CH, Wong L, et al: Denaturing gradient gel electrophoresis profiles of bacteria from the saliva of twenty four different individuals form clusters that showed no relationship to the yeasts present. Arch Oral Biol 2017;82:6-10.

8 Weerasekera MM, Wijesinghe GK, Jayarathna TA, et al: Culture media profoundly affect Candida albicans and Candida tropicalis growth, adhesion, and biofilm development. Mem Inst Oswaldo Cruz 2016;111:697-702.

9 Mukherjee PK, Zhou G, Munyon R, et al: Candida biofilm: a well-designed protected environment. Med Mycol 2005;43:191-208. 
10 Bizerra FC, Nakamura CV, De Poersch C, et al: Characteristics of biofilm formation by Candida tropicalis and antifungal resistance. FEMS Yeast Res 2008;8:442-450.

11 Jin Y, Samaranayake LP, Samaranayake YH, et al: Biofilm formation of Candida albicans is variably affected by saliva and dietary sugars. Arch Oral Biol 2004;49:789-798.

12 Parahitiyawa NB, Samaranayake YH, Samaranayake LP, et al: Interspecies variation in Candida biofilm formation studied using the Calgary biofilm device. APMIS 2006;114: 298-306.

13 Loesche WJ: Microbiology of dental decay and periodontal disease; in Baron S (ed): Medical Microbiology, ed 4. Galveston, University of Texas Medical Branch at Galveston, 1996, p 99.

14 Ibrahim OO: High intensity sweeteners chemicals structure, properties and applications. NSD 2015;1:88-94.

15 Mattes RD, Popkin BM: Nonnutritive sweetener consumption in humans: effects on appetite and food intake and their putative mechanisms. Am J Clin Nutr 2009;89:1-14.

16 Johnston CA, Stevens B, Foreyt JP: The role of low-calorie sweeteners in diabetes. US Endocrinol 2013;9:96-98.
17 Okpalugo J, Ibrahim K, Inyang US: Toothpaste formulation efficacy in reducing oral flora. Trop J Pharm Res 2009;8:71-77.

18 Marsh PD, Bradshaw DJ: Physiological approaches to the control of oral biofilms. Adv Dent Res 1997;11:176-185.

19 American Type Culture Collection: ATCC ${ }^{\circledR}$ : The essentials of life science research. 2017. https://www.atcc.org/Search_Results.aspx?d $\mathrm{sNav}=\mathrm{Ntk}:$ PrimarySearch $\% 7 \mathrm{cmi} \% 7 \mathrm{c} 3 \% 7 \mathrm{c}, \mathrm{N}$ $y:$ True,Ro:0,N:1000552\&searchTerms $=$ mi\&r edir $=115.10 .2017$.

20 Tsang PW, Bandara HMHN, Fong W: Purpurin suppresses Candida albicans biofilm formation and hyphal development. PLoS One 2012;7:1-8.

21 Serrano-Fujarte I, López-Romero E, ReynaLópez GE, et al: Influence of culture media on biofilm formation by Candida species and response of sessile cells to antifungals and oxidative stress. Biomed Res Int 2015;2015: 783639.

22 Forssten SD, Björklund M, Ouwehand AC: Streptococcus mutans, caries and simulation models. Nutrients 2010;2:290-298.

23 McCourtie J, Douglas LJ: Relationship between cell surface composition of Candida albicans and adherence to acrylic after growth on different carbon sources. Infect Immun 1981;32:1234-1241.
24 Nassar HM, Li M, Gregory RL: Effect of honey on Streptococcus mutans growth and biofilm formation. Appl Environ Microbiol 2012;78:536-540.

25 Waldrop R, McLaren A, Calara F, et al: Biofilm growth has a threshold response to glucose in vitro. Clin Orthop Relat Res 2014;471: 3305-3310.

26 Abu-Elteen KH: The influence of dietary carbohydrates on in vitro adherence of four Candida species to human buccal epithelial cells. Microb Ecol Health Dis 2005;17:156-162.

27 Modrzewska B, Kurnatowski P: Adherence of Candida sp. to host tissues and cells as one of its pathogenicity features. Ann Parasitol 2015; 61:3-9.

28 Shrout JD, Chopp DL, Just CL, et al: The impact of quorum sensing and swarming motility on Pseudomonas aeruginosa biofilm formation is nutritionally conditional. Mol Microbiol 2006;62:1264-1277.

29 Meurman JH, Siikala E, Richardson M, et al: Non-Candida albicans Candida yeasts of the oral cavity; in Méndez-Vilas A (ed): Communicating Current Research and Educational Topics and Trends in Applied Microbiology. Badajoz, Formatex, 2007, pp 719-731. 